\title{
New challenges and mitigation strategies for resident selection during the coronavirus disease pandemic
}

Dear Editor,

The coronavirus disease (COVID-19) pandemic has presented challenges to resident education, and we foresee a unique challenge in the residency selection for emergency medicine (EM) programs. There are approximately 120 applicants to the Royal College of Physicians and Surgeons of Canada program and 120 to the College of Family Physicians of Canada EM program each year at our institution (personal communication from University of Ottawa program directors). Programs will be forced to deviate from conventional norms in the resident selection process. With some foresight, most of these challenges can be mitigated. ${ }^{1}$

Most teaching hospitals in Canada have either greatly limited or completely halted visiting elective students and residents. This poses a number of issues to the programs, as well as the candidates, which we feel can be classified under two main categories:

- Inability to experience environment at the residency training location (hospital and city)

- Inability to have face-to-face time with program directors, residents, and faculty from the program of interest

When selecting EM programs, prospective trainees surveyed place great value in their personal experience with residents and faculty in program selection. ${ }^{2}$ In another resident candidate survey, subjective considerations, such as happy residents, program personality, and faculty enthusiasm, were the top factors in selecting their EM residency program. ${ }^{3}$ With the limitations to visiting electives, these challenges will need to be tackled in a creative manner. Our programs are looking to virtual platforms as a way to mitigate these challenges in the following manner:

Inability to experience environment at the residency training location

1. Online video highlighting the program's city (EMOttawa video): https://youtu.be/EpCoiki42YE

2. Resident-led blog on residency experiences (emottawablog.com Resident Insights): https://emottawablog.com/category/residentinsights/

3. Video tours of non-patient care areas: short video highlighting teaching areas, simulation centre

Inability to have face-to-face time with program directors, residents, and faculty

1. Program directors, faculty, alumni, and resident introduction videos (These short videos will highlight some members of the residency program and provide insights to the program's features and unique qualities.)

2. "Ask Me Anything" sessions with program directors and chief residents (These online sessions using social media platforms, such as Twitter $\AA$, to ensure potential residents get to ask the questions they would like answered prior to their application submission and interviews. One session is planned prior to each of these key dates.)

3. One-on-one virtual meetings with program directors with any interested prospective resident

Other potential mitigation strategies

1. Early preparation for challenges of virtual interviews that will allow for all travel and social distancing protocols to be adhered to ${ }^{4}$

2. Virtual social night prior to the interview day to allow for an informal meeting of candidates with the interview panel

3. Updated department website to highlight the program and link to new resources being offered

4. Use of established social media platforms to highlight program

Correspondence to: Dr. Hans Rosenberg, 1053 Carling Ave., Ottawa, ON K1Y 4E9, Canada; Email: hrosenberg@ toh.ca.

(c) Canadian Association of Emergency Physicians 2020

CJEM 2020;22(6):1-3

DOI 10.1017/cem.2020.454 


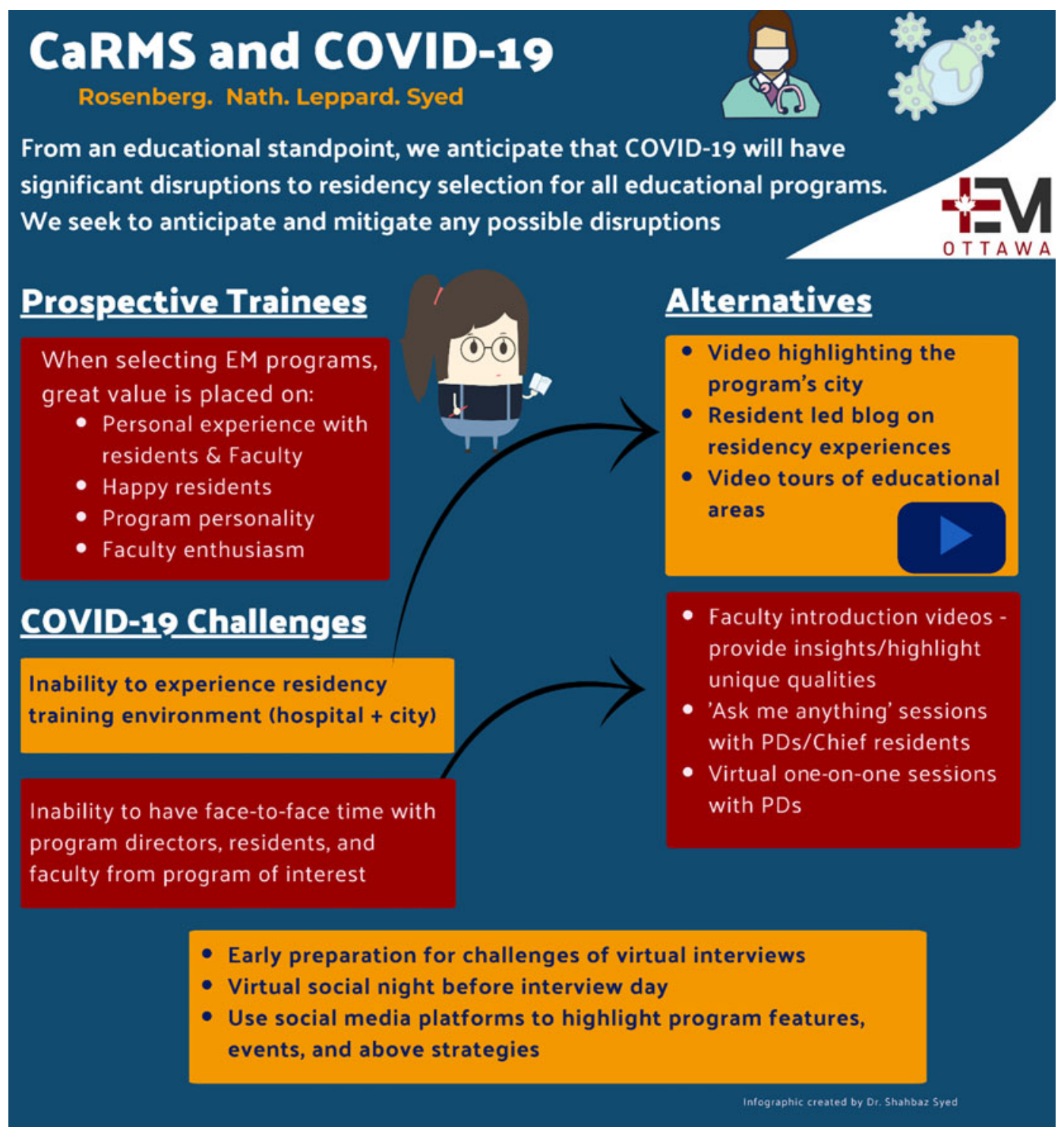

Figure 1. Challenges and mitigation strategies for resident selection during the COVID-19 Pandemic.

features, events, and previously mentioned strategies

The COVID-19 pandemic has caused a disruption to the classic postgraduate trainee selection process. Mitigation strategies described previously will allow potential applicants to experience some of the features that make programs unique and often lead to their choice of program (Figure 1). Furthermore, these strategies can be used in the future in conjunction with more classic face-to-face encounters and electives to ensure the best program fit for prospective residency candidates.

\section{Hans Rosenberg, MD}

Avik Nath, MD, MScPH

Jennifer Leppard, MD

Shahbaz Syed, MD
Department of Emergency Medicine, University of Ottawa, Ottawa, ON

Keywords: COVID-19, education, medical students, pandemic, residents and fellow

Competing interests: None declared.

\section{REFERENCES}

1. Hammoud M, Standiford T, Carmody J. Potential implications of COVID-19 for the 2020-2021 residency application cycle. $7 A M A$ 2020;324(1):29-30. 
2. Love J, Howell J, Hegarty C, et al. Factors that influence medical student selection of an emergency medicine residency program: implications for training programs. Acad Emerg Med 2012;19(4):455-60.
3. Yarris LM, Deiorio NM, Lowe RA. Factors applicants value when selecting an emergency medicine residency. West $\mathcal{f}$ Emerg Med 2009;10 (3):159-62.
4. Jones $\mathrm{R}$, Abdelfattah $\mathrm{K}$. Virtual interviews in the era of COVID-19: a primer for applicants. I Surg Educ 2020;77(4):733-4. 